\title{
Recognizing immunodeficiency in children with recurrent infections: What are the predictive factors?
}

\author{
Murat Sütçü, Manolya Acar, Hacer Aktürk, Selda Hançerli-Törün, Nuran Salman, \\ Ayper Somer \\ Divisions of Pediatric Infectious Diseases and Clinical Immunology, Istanbul University Istanbul Faculty of Medicine, \\ Istanbul,Turkey.E-mail: sutcu13@yahoo.com, murat.sutcu@istanbul.edu.tr \\ Received: 2nd December 2016, Revised: 4th February 2017, Accepted: 7th February 2017
}

SUMMARY: Sütçüi M, Acar M, Aktürk H, Hançerli-Törün S, Salman N, Somer A. Recognizing immunodeficiency in children with recurrent infections: What are the predictive factors?

The aim of this study was to evaluate the children presenting with the complaint of recurrent infections and to determine the possible predictive factors foreseeing the requirement for further investigations. Turk J Pediatr 2016; 58: 609-615.

This study is a retrospective analysis of 507 children (221 female, $43.6 \%$ ) with median age of 46 (range 4-190) months, who attended our department with the complaint of recurrent infections between January 2013 and December 2014 during two years period.

The majority of the patients were preschool age children (n: 360, 71\%). Most of the infections were upper repiratory tract infections [URTI (n: 380, 75\%)]. The patients were divided into 4 diagnostic groups; as atopic children (n: 148, 29.2\%), children with primary immune deficiency [PID (n: 54, 10.7\%)], patients with chronic disorders (n: 40, 7.9\%) and the majority, healthy subjects ( $\mathrm{n}: 265,52.3 \%$ ). Among school age patients, the incidence of atopic children was significantly high (p: 0.016). Presenting at adolescent age group, growth retardation and hypogammaglobulinemia were the predictive risk factors for PID. Antibody (B cell) deficiencies (n: 43, 80\%), and among those, selective IgA deficiency ( $\mathrm{n}: 23,4.5 \%$ ) constituted the majority of the patients in PID. Rheumatological diseases ( $\mathrm{n}:$ 9, 1.8\%), mainly periodic fever syndromes, were the most common chronic disorders. Malignancy (acute lymphoblastic leukemia and lymphoma) was obtained in two patients $(0.4 \%)$.

Complaint of recurrent infections is very common among children attending health care facilities. It is crucial for clinicians to differentiate the children who need further investigations, urgent medical attention.

Key words: children, immunodeficiency, recurrent infections.

General practitioners and pediatricians often come across with children who are presented with the complaint of recurrent infections. Although families and primary caregivers worry about the underlying serious disorders, aproximately half of those children are completely healthy where as one third of them are atopic ${ }^{1}$. The remaining $20 \%$ comprise the primary immune deficiencies (PIDs) and the chronic disorders. Reccurent infections are the main features of the childhood presentation of PIDs. The primary duty of the physicians is to differentiate the minority of these children requiring prompt diagnosis and treatment from those healthy and the atopic ones.

Another confusing issue for both the practitioners and the parents is the definiton of the "recurrent infections", since healthy children, especially the ones attending kindergarden or school may encounter up to 10 to 12 respiratory tract infections (RTIs) per year. Recurrent infections may be generally defined as two or more severe infectious episodes in a year, three or more respiratory infections (eg, sinusitis, otitis, bronchitis) in one year, or the requirement of antibiotics for two months 
per year. On the other hand, the term "severe infections" includes infections with uncommon pathogens and/or uncommon complications, inefficient treatment with oral antibiotics requiring parenteral therapy, fever lasting more than a week and persistent laboratory abnormalities as acute phase reactants and imaging.

The Jeffrey Modell Foundation has created the "10 warning signs of PIDs" in the early life $^{2}$ (Table I). In general, those signs are the practical summary of the definitions for recurrent and severe infections. In order to evaluate a child presenting with recurrent infections, a clinician should perform a detailed history and physical examination in addition to the appropriate laboratory tests. In this concept, the strategy of cost effectiveness and the performance of minimum required tests are feasible.

Our department is a pediatric infectious disease and immunology outpatient clinic, which is both a referral center and a facility with open public access. So, the profile of our patients represent both the properties of the ones attending to primary care physicians and general pediatricians in addition with the selectively referred immune deficient ones. In this study, our aim was to investigate the clinical characteristics of children attending our clinic with the complaint of recurrent infections and to analyze the predictive risk factors in order to select the patients with possible immunodeficiencies.

\section{Material and Methods}

Study patients were selected among those admitting to outpatient clinic of pediatric infectious disease and immunology department between January 2013 and December 2014. Pediatric patients (age range: 0-18 years) presented with following complaints and/ or medical history were enrolled: $\geq 8$ upper respiratory tract infections (URTIs) per year, $\geq 2$ pneumonias or serious sinus infections within one year, $\geq 4$ new ear infections within 1 year, $\geq 2$ episodes of sepsis or meningitis in a lifetime, use of antibiotics more than 2 months with little effect. The patients with known congenital disorders, chronic diseases or proven immune deficiency at the time of presentation were excluded.

Medical records of 507 children fulfilling the inclusion criteria were retrospectively reviewed regarding the following clinical and laboratory variables: age of symptom onset, paternal consanguinity, family history of recurrent infections, household exposure to smoke, use of nursing bottle, duration of breastfeeding, presence of a sibling with day-care or school attendance, complete blood count, routine blood chemistry, C-reactive protein (CRP), erythrocyte sedimentation rate (ESR), and serum immunoglobulin (Ig) levels (IgG, IgA, IgM, IgE). In addition, results of IgG subgroup and lymphocyte subgroup analysis, serological tests, skin prick test, sweat chloride test, tuberculin skin test (TST) and radiological examinations which had been performed in selective cases were also recorded. Serum Ig and IgG subgroup analyses were performed by nephelometrical method (Date Behring Marburg Gmbh, Germany) and compared with agerelated normal values. Lymphocyte subgroup

Table I. Warning Signs of Primary Immune Deficiency.

Four or more new ear infections within 1 year

Two or more serious sinus infections within 1 year

Two or more months on antibiotics with little effect

Two or more pneumonias within 1 year

Failure of an infant to gain weight or grow normally

Recurrent, deep skin or organ abscesses

Persistent thrush in mouth or fungal infections on skin

Need for intravenous antibiotics to clear infections

Two or more deep-seated infections including septicemia

A family history of primary immune deficiency 
analysis was made by four-color flow cytometry (BD Facs Calibur, BD Calibur, BD Biosciences, San Jose, California, USA) using CD45FITC, CD3FITC, CD4PE, CD8APC, CD19FITC and CD16-56 PE monoclonal antibodies and compared with age related normal values using the normal levels of Turkish children previously published by Aksu et al. ${ }^{3}$.

After a thorough clinical and laboratory evaluation, the patients were grouped into four different diagnostic categories according to following criteria: (i) "PIDs", the patients who met diagnostic criteria determined by ESID ${ }^{4}$; (ii) chronic disorders (CD), the patients who were diagnosed with a nonimmune chronic disease or a congenital defect after their evaluation for recurrent infections; (iii) atopic children (AC), the patients who were defined as atopic due to results of spesific IgE and/or skin prick tests; (iv) "healthy subjects (HS)", the patients with no underlying pathology that could be defined.

Since age is an important determining factor in pediatric population, the study population was divided into four subgroups according to age: infants $(<1$ years of age), preschool-aged children (between 1-5 years of age), school-

Table II. Some Clinical and Laboratory Features of the Children Presenting with Frequent Infections According to Final Diagnostic Groups.

\begin{tabular}{|c|c|c|c|c|c|}
\hline & $\begin{array}{c}\text { HS } \\
\text { N: } 265 \\
(52.3 \%)\end{array}$ & $\begin{array}{c}\mathrm{AC} \\
\mathrm{N}: 148 \\
(29.2 \%)\end{array}$ & $\begin{array}{c}\text { PID } \\
\text { N: } 54 \\
(10.7 \%)\end{array}$ & $\begin{array}{c}\text { CD } \\
\mathrm{N}: 40 \\
(7.9 \%)\end{array}$ & $\begin{array}{l}\text { Total } \\
\text { N: } 507\end{array}$ \\
\hline \multicolumn{6}{|l|}{ Age distribution, n (\%) } \\
\hline Infant & $14(5.3)$ & $6(4.1)$ & $10(18.5)^{*}$ & $2(5.0)$ & $32(6.3)$ \\
\hline Preschool age & $198(74.7)$ & $99(66.9)$ & $33(61.1)$ & $30(75)$ & $360(71.0)$ \\
\hline School age & $49(18.5)$ & $40(27.0)^{\mathbb{S}}$ & $7(13.0)$ & $7(17.5)$ & $103(20.3)$ \\
\hline Adolescent age & $4(1.5)$ & $3(2.0)$ & $4(7.4)^{\mathbb{S}}$ & $1(2.5)$ & $12(2.4)$ \\
\hline $\begin{array}{l}\text { Duration of complaints } \\
\text { (months), mean } \pm \text { SD }\end{array}$ & $\begin{array}{l}23.24 \pm \\
20.35\end{array}$ & $\begin{array}{l}29.95 \pm \\
20.93^{*}\end{array}$ & $\begin{array}{l}23.03 \pm \\
20.99\end{array}$ & $\begin{array}{l}21.65 \pm \\
17.31^{\mathbb{S}}\end{array}$ & $\begin{array}{l}25.05 \pm \\
21.95\end{array}$ \\
\hline \multicolumn{6}{|c|}{ Type and frequency of infections, $\mathrm{n}(\%)$} \\
\hline$\geq 8 \mathrm{RTI} /$ year & $175(61.4)^{*}$ & $69(46.6)^{\mathbb{S}}$ & $19(35.2)$ & $22(55.0)$ & $285(56.2)$ \\
\hline $\begin{array}{l}\geq 2 \text { serious sinus/ } \\
\text { pulmonary infection/year }\end{array}$ & $48(18.1)$ & $59(39.9)^{*}$ & $19(35.2)$ & $14(35)$ & $140(27.6)$ \\
\hline$\geq 4$ new ear infection/year & $13(4.9)$ & $5(3.4)$ & $2(3.7)$ & $2(5.0)$ & $22(4.3)$ \\
\hline $\begin{array}{l}\geq 2 \text { sepsis/ meningitis } \\
\text { lifetime infection }\end{array}$ & $7(2.6)$ & $1(0.7)$ & $5(9.3)^{\S}$ & $1(2.5)$ & $14(2.8)$ \\
\hline $\begin{array}{l}\geq 2 \text { mo. of antibiotics } \\
\text { with minor effect }\end{array}$ & $22(8.3)$ & $14(9.5)$ & $9(16.7)^{\mathbb{S}}$ & $1(2.5)$ & $46(9.1)$ \\
\hline \multicolumn{6}{|l|}{ Laboratory, n (\%) } \\
\hline Lymphopenia & $8(3.0)$ & $5(3.4)$ & $5(9.3)$ & $4(10.3)$ & $22(4.3)$ \\
\hline Neutropenia & $9(3.4)$ & $4(2.7)$ & $4(7.4)$ & $3(7.7)$ & $20(3.9)$ \\
\hline Anemia & $14(5.3)$ & $4(2.7)$ & $6(11.3)$ & $8(20.5)^{*}$ & $32(6.3)$ \\
\hline Increased ESR & $87(34.9)$ & $63(44.7)$ & $21(39.6)$ & $22(57.9)$ & $193(38.1)$ \\
\hline Hypogammaglobulinemia & $1(0.4)$ & 0 & $12(22.2)^{*}$ & $5(12.5)^{*}$ & $18(3.6)$ \\
\hline
\end{tabular}

AC: atopic child; CD: chronic disease; HS: healthy subjects; PID: primary immune deficiency; SD: standard deviation; * $\mathrm{p}<0.001$ "Fisher chi-square", ${ }^{\mathbb{p}} \mathrm{p}<0,05$ "Fisher chi-square". 
aged children (between 6-11 years of age) and adolescents (12-18 years of age).

\section{Statistical analysis}

Statistical analysis of data was performed with the statistical package for social science (SPSS) for Windows version 21.0 (SPSS 21.0, SPSS Inc. USA). Categorical and numerical variables between groups were compared by using "Fisher chi-square" and "independent sample $\mathrm{t}$ tests," respectively. Statistical significance limit was accepted as $p<0.05$. Logistic regression analysis was performed for the data with $\mathrm{p}$ values $<0.05$.

The research project has received the confirmation of the Local Ethical Committee of Istanbul University, Istanbul Medical Faculty (03-2016). All children and their parents were informed about the details of the study and written consent was obtained from the parents of the children involved.

\section{Results}

Five hundred and seven patients (221 female, $43.6 \%$ ) with a median age of 46 months (4-190) were enrolled. Majority of the patients were preschool-aged children $(n=360,71 \%)$ followed in the frequency by school-aged children, infants and adolescents (Table II). Approximately half of the children (n: 265, 52.3\%) were accepted as healthy subjects, since no pathology could be determined after a thorough evaluation. In the other half, AC constituted the major group ( $\mathrm{n}: 148,29.2 \%)$. The patients defined as PID and $\mathrm{CD}$ were less frequent (n: $54,10.7 \%$ and n: $40,7.9 \%$, respectively).

Congenital malformations [congenital heart disease (n: $2,0.4 \%)$, congenital renal abnormality ( $\mathrm{n}: 2,0.4 \%$ ), cystic adenoid malformation $(\mathrm{n}: 1,0.2 \%)]$, neurological disorders [spinal muscular atrophy (n: 2,
$0.4 \%$ ) and others (n: $6,1.2 \%$ )], rheumatological diseases [periodic fever aphthous stomatitis pharyngitis and adenitis (PFAPA, n: 3, 0.6\%), familial mediterranean fever (FMF, n: $5,1 \%$ ), juvenile idiopathic arthritis ( $\mathrm{n}: 1,0.2 \%)$ ], metabolic disorders (n: 3, 0.6\%), chronic pulmonary disease [bronchopulmonary displasia (n: $2,0.4 \%$ ) and cystic fibrosis (n: 2, 0.4\%)], gastroesophageal reflux (n: $4,0.8 \%$ ), systemic infections [tuberculosis (n: $4,0.8 \%$ ) and human immunodeficiency virüs infection (n: $1,0.2 \%)]$ and malignancy [acute leukemia (n: $1,0.2 \%$ ) and lymphoma (n: $1,0.2 \%)]$ constituted the chronic disorders.

When diagnostic groups were classified according to age, the incidence of PID in infants and adolescents were significantly higher than preschool and school-aged children $(\mathrm{p}<0.001$ and 0.03 , respectively). On the other hand, the incidence of atopy was significantly higher (p: 0.016) in school-aged children (Table II).

\section{Presentation}

Mean duration of symptoms at the time of presentation was $25.05 \pm 21.95$ months. It was found to be longer in AC group and shorter in CD group (Table II). The most common presenting complaint was recurrent RTIs $(56.2 \%$ of the patients), and was noted to be more frequent in HS and AC group ( $\mathrm{p}$ $<0.001$ and 0.005 , respectively). Most of the RTIs were URTIs (n: $285,56.2 \%$ ). The patients diagnosed as PID had more frequently suffered from sepsis/meningitis and unresponsiveness to long-term antibiotic treatment $(p=0.002$ and 0.04 , respectively). Previous history of hospitalization $(24.3 \%$ in all patients) was more frequent in

PID and CD groups (48.1\%, p $<0.001$ and $45 \%, p=0.001$ respectively). Thirty-seven patients $(7.3 \%)$ presented with somatic growth retardation, which was more frequent in PID

Table III. Multivariate Analysis of Predictive Risk Factors for Identification of Primary Immune Deficiency Among Children Presenting with Recurrent/Frequent Infections.

\begin{tabular}{lcc}
\hline Variables & OR $(95 \% \mathrm{CI})$ & $\mathrm{p}$ \\
\hline Adolescent age & $4.4(1.18-16.81)$ & 0.027 \\
Growth retardation & $2.5(1.02-6.43)$ & 0.045 \\
Hypogammaglobulinemia & $9.1(2.68-30.3)$ & $<0.001$ \\
\hline
\end{tabular}

CI: confidence interval; OD: Odds ratio 
and CD groups $(24.1 \%$ and $35 \%$ respectively, $\mathrm{p}<0.001$ in both).

\section{Risk factors}

Positive family history of recurrent infections was determined in 232 patients (45.8\%), without any significant difference among the groups. Paternal consanguinity was declared in $12.6 \%$ of the study patients with a significantly higher frequency in PID group $(27.8 \%, \mathrm{p}<0.001)$. Mean duration of breastfeeding were $11.59 \pm$ 7.23 months in all study patients and did not differ among the groups. Household exposure to smoke was common ( $54.8 \%$ of all patients) and the patients in HS and AC groups were found to be more frequently exposed than other groups $(\mathrm{p}=0.028$ and $\mathrm{p}=0.007$, respectively). Prematurity was present in $4.9 \%$ of the all patients, with a significantly higher frequency in CD group $(25 \%, \mathrm{p}<0.001)$. There was no difference among groups regarding bottle feeding (n: 201/571; 25\%). The incidence of attending kindergarden was statistically more common among the subgroup of children that were between 3-6 years of age (n: 77, 42.5\%, $\mathrm{p}=0.009$ ).

\section{Laboratory evaluation}

Twenty-two (4.3\%) patients presented with lymphopenia and 20 (3.9\%) children with neutropenia. Hypogammaglobulinemia was observed in 18 (3.6\%) children, all of whom were grouped as either PID (13/54) or CD $(5 / 40)$. Increased erythrocyte sedimentation rate, which was present in $38.1 \%$ of all patients was more frequently detected in $\mathrm{CD}$ group (57.9\%, $\mathrm{p}=0.02)$.

\section{The patients with PID}

Detailed immunological evaluation including IgG subgroup analysis and lymphocyte subgroup analyses were performed in 135 $(26.6 \%)$ patients who were suspected to be immune deficient. Fifty-four $(10.7 \%)$ children were diagnosed as PID according to ESID criteria 4 : primary antibody deficiency (PAD) in 43 patients $(80 \%)$, combined immunodeficiency (immunodeficiency affecting cellular and humoral immunity) in 8 patients $(14.8 \%)$ and congenital phagocytic defects in 3 patients $(5.5 \%)$. Selective IgA deficiency was the most commonly defined immunological disorder (n: $23,4.5 \%$ of all patients). In order to determine predictive factors for a final diagnosis of
PID in a child presenting with a history of frequent infections, a multivariate analysis was performed. Presentation in adolescence, growth retardation and hypogammaglobulinemia were found to be the predictive risk factors for PID (Table III).

\section{Discussion}

In this study, a large cohort of children presenting with frequent/recurrent infections were evaluated regarding clinical and laboratory features, risk factors and final diagnoses. At the final asessment, approximately half of these children were healthy subjects without underlying disorders that could be identified with our current medical knowledge. The majority of the children in the other half were determined to be atopic. Primary immune deficiency and CD were diagnosed in $\leq 10 \%$ of the patients for each. These incidences are quite similar to the formal information in this issue ${ }^{1,5}$.

A child, depending on age, can experience 4-8 times of RTIs in a year ${ }^{6,7}$. Moreover, the children with possible risk factors like having older siblings or attending day care centers may experience up to 10-12 URTIs ${ }^{8,9}$. The same was also true for our study cohort. The incidence of children attending kindergarden was significantly more common. So, more determining risk factors other than number of infectious episodes should be considered when a child is being evaluated for possible PID. In our study, we observed that PID was more frequently the final diagnosis in patients with the history of serious bacterial infections and the requirement for parenteral antibiotics.

Immune system maturation persists during infancy. This phenomenon by itself may explain recurrent infections. However, children below 12 months of age should be evaluated carefully, since many of the PID syndromes may present at this age group. Consistently, we found that frequency of PID was significantly high in infants. The same was also true for the adolescent age group. Since, children with common variable immune deficiency may have their onset in those ages, and many other chronic illnesses and medications may cause secondary immunosuppression, special attention should be given to adolescent patients ${ }^{10}$.

Children exposed to risk factors like crowded 
population, cigarette smoke and airborne allergens may experience more frequent RTIs ${ }^{11-}$ 14. Tagliacarne et al. ${ }^{15}$, showed increased immunglobulin production in adenoid tissue of atopic children exposed to smoke. More than half of our study population declared exposure to household smoke. It was more frequently reported in $\mathrm{HSs}$ and $\mathrm{AC}$, consistent with the mentioned data. The most important place for a child to face with airborne pathogens other than smoke is the time spent at school. Thus, AC mainly experience more often and longer periods of RTIs when compared with nonatopic classmates. In some cases, allergic airway symptoms may be misdiagnosed as RTIs. In fact, it is crucial to differentiate the present situation, whether it is a new infection or exacerbation of underlying atopy, since the treatment options differ. Besides, PIDs may also coexist with atopic disorders ${ }^{5}$. The type and the severity of the infection may be a clue in that case. According to our results, AC mostly experienced RTIs, however, patents with PID suffered from more severe infections like sepsis or meningitis.

Initial laboratory investigations are useful to predict the need for further evaluation. For instance, lymphopenia and hypogammaglobulinemia should warn a physician for possible PID, as well as anemia and increased sedimentation rate may be a clue for underlying CDs. The frequency of anemia and increased ESR was significantly higher in patients with CDs, and the number of patients presented with hypogammaglobulinemia were both high in PID and CD groups. Contrary to well-known association between lymphopenia and immune deficiency, lymphopenia was not more frequent in the PID group. This may be explained by the transient lymphopenia observed secondary to a recent infection within the other groups.

The incidence of PID was $10.7 \%$ (n: 54) in our study, in consistence with the previous studies $^{16}$. Among those children, PAD; mainly selective IgA deficiency constituted the majority. Combined $\mathrm{B}$ and $\mathrm{T}$ cell deficiencies and other PID disorders were less frequent. Paternal consanguinity incidence was significantly higher in children with PID. This finding is not surprising since PIDs may be inherited in autosomal recessive manner or represent familial tendency ${ }^{17}$. In countries with high incidence of paternal consanguinity, like ours, clinicians should pay special attention to family history in terms of prior immune deficient relative and unexplained death of a sibling.

Recurrent infections may be the first manifestation in children with chronic diseases like congenital abnormalities. On the other hand, some other disorders, like periodic fever syndromes may be mistaken by both families and clinicians as having recurrent infections ${ }^{18}$. Five patients $(1 \%)$ were diagnosed with FMF while 3 children $(0.6 \%)$ were detected to have PFAPA syndrome in our study population. Rarely, childhood malignancies can be presented as recurrent infections since malignant disorders create secondary immunosuppression. In our study two 2 children, presented with recurrent infections, had final diagnoses of acute lymphoblastic leukemia and lymphoma.

Multivariate analysis revealed some independent risk factors for making a final diagnosis of PID in a patient presenting with recurrent infections. Being adolescent at presentation was found to increase the probability of PID for 4.4 times. Other significant predictive risk factors were somatic growth retardation (2.5 times) and presence of hypogammaglobulinemia (9 times). These findings can be important clues for clinicians to differentiate a patient with PID.

The evaluation of a high number of children presented with frequent/recurrent infections revealed that, the majority of these children were otherwise healthy and a substantial number of them were atopic. The incidence of PID and a broad range of underlying CDs were less often. In the current study, presenting age, medical history, exposure to some risk factors, physical examination findings and first level laboratory tests were found to be important marks in guiding physicians to differentiate these children.

\section{REFERENCES}

1. Stiehm ER. Approach to the child with recurrent infections. UpToDate, 2014.

2. 10 Warning Signs: Jeffrey Modell Foundation. Available at: www.jmfworld.com/library/educationalmaterials/10-warning-signs. Accessed December, 2016. 
3. Aksu G, Genel F, Koturoglu G, et al. Serum immunoglobulin (IgG, IgM, IgA) and IgG subclass concentrations in healthy children: a study using nephelometric technique. Turk J Pediatr 2005; 47: 19-24.

4. Clinical Diagnostic Criteria for Primary Immunodeficiencies. Available at: https://esid.org/ Working-Parties/Clinical/Resources/Diagnostic-criteriafor-PID2. Accessed December, 2016.

5. MacGinnitie A, Aloi F, Mishra S. Clinical characteristics of pediatric patients evaluated for primary immunodeficiency. Pediatr Allergy Immunol 2011; 22: 671-675.

6. Monto AS. Viral respiratory infections in the community: epidemiology, agents, and interventions. Am J Med 1995; 99: 24-27.

7. Campbell H. Acute respiratory infection: a global challenge. Arch Dis Child 1995; 73: 281-283.

8. Janson C. The effect of passive smoking on respiratory health in children and adults. Int J Tuberc Lung Dis 2004: 8: 510-516.

9. Grüber C, Keil T, Kulig M, et al. History of respiratory infections in the first $12 \mathrm{yr}$ among children from a birth cohort. Pediatr Allergy Immunol 2008; 19: 505512 .

10. Hogan MB, Wilson NW. Common variable immunodeficiency in children. Up To Date; 2014.
11. Marseglia GL, Avanzini MA, Caimmi S, et al. Passive exposure to smoke results in defective interferongamma production by adenoids in children with recurrent respiratory infections. J Interferon Cytokine Res 2009; 29: 427-432.

12. Lugade AA, Bogner PN, Thatcher THJ, et al. Cigarette smoke exposure exacerbates lung inflammation and compromises immunity to bacterial infection. Immunol. 2014; 192: 5226-5235.

13. Janson C. The effect of passive smoking on respiratory health in children and adults. Int J Tuberc Lung Dis 2004: 8: 510-516.

14. Gaffin JM. Postnatal environmental tobacco smoke exposure is associated with objective markers of atopy in preschool-aged children. Evid Based Med 2015; 20: 219-221.

15. Tagliacarne SC, Valsecchi C, Castellazi AM, et al Impact of passive smoke and/or atopy on adenoid immunoglobulin production in children. Immunol Lett 2015; 165: 70-77.

16. Stiehm ER. The four most common pediatric immunodeficiencies. J Immunotoxicol 2008; 5: 227-234.

17. Bonilla FA, Khan DA, Ballas ZK, et al. Practice parameter for the diagnosis and management of primary immunodeficiency. J Allergy Clin Immunol 2015; 136: 1186-1205.

18. Kasapçopur Ö, Arısoy N. Ailesel Akdeniz Ateși ve diğer otoenflamatuar hastalıklar. Türk Pediatri Arşivi 2006; 41: 9-17. 